\title{
Retrograde amnesia for visual memories after hippocampal damage in rats
}

\author{
Jonathan Epp, ${ }^{1,4}$ Julian R. Keith, ${ }^{2,3}$ Simon C. Spanswick, ${ }^{1}$ Jared C. Stone, ${ }^{1}$ \\ Glen T. Prusky, ${ }^{1}$ and Robert J. Sutherland ${ }^{1}$ \\ ${ }^{1}$ Canadian Centre for Behavioural Neuroscience, University of Lethbridge, Lethbridge, Alberta T1K $3 \mathrm{M4}$, Canada; ${ }^{2}$ Department \\ of Psychology, University of North Carolina, Wilmington, North Carolina 28403, USA; ${ }^{3}$ Memory Assessment and Research \\ Services (MARS), Wilmington, North Carolina 28405, USA
}

\begin{abstract}
It is generally believed that the hippocampus is not required for simple discrimination learning. However, a small number of studies have shown that hippocampus damage impairs retention of a previously learned visual discrimination task. We propose that, although simple discrimination learning may proceed in the absence of the hippocampus, it plays an important role in this type of learning when it is intact. In order to test the role of the hippocampus in simple discrimination learning, we performed a series of experiments utilizing a two-choice picture discrimination task. Our experiments confirm that rats readily learn simple two-choice picture discriminations after hippocampus damage. However, if such discriminations are first learned while the hippocampus is intact, subsequent hippocampus damage causes severe retrograde amnesia for the discriminations. Furthermore, retrograde amnesia for simple picture discriminations was equally severe when the interval between training and damage was $1 \mathrm{~d}$ or $60 \mathrm{~d}$; remote picture memories are not spared. Similarly, the rule or schema underlying a recently or remotely acquired picture discrimination learning set was lost after hippocampus damage. The severity of retrograde amnesia for simple picture discriminations is negatively correlated with the volume of spared hippocampus tissue. Thus, the hippocampus plays an essential role in long-term memories supporting simple picture discriminations.
\end{abstract}

Much of what is known about the role of the hippocampus in learning and memory comes from studies of anterograde amnesia after hippocampus damage. There is a consensus that the ability to learn simple discrimination tasks typically is not affected by anterograde amnesia (Broadbent et al. 2007). In simple discrimination tasks, usually involving many trials of training, one stimulus is reinforced and another is not. As such, simple discriminations are among the class of problems that have linear solutions (i.e., during training, $\mathrm{S}+$ acquires greater excitatory associative strength than $S-$ ). Numerous experiments have confirmed that anterograde amnesia after hippocampus damage does not affect simple discrimination problems (Sutherland and Rudy 1989; Whishaw and Tomie 1991; Alvarado and Rudy 1995). Thus, data from studies of anterograde amnesia after hippocampus damage support the conclusions that the hippocampus is not necessary for simple discrimination learning and that neural systems outside the hippocampus are capable of encoding, storing, and retrieving memories supporting simple discrimination learning.

One might conclude from the foregoing that the learning systems that support simple discriminations also function independently of the hippocampus when the brain is intact. Alternatively, even though simple discrimination learning is possible when the hippocampus is not available, the hippocampus may still participate in learning simple discriminations when it is available (McDonald et al. 2007). Indeed, a small number of studies supply evidence that if simple discriminations are learned initially while animals have an intact hippocampus, subsequent damage to the hippocampus causes retrograde amnesia. Sara (1981) first reported retrograde amnesia in rats after hippocam-

\footnotetext{
${ }^{4}$ Corresponding author.
}

E-mail jonepp@interchange.ubc.ca; fax (604) 822-6923.

Article is online at http://www.learnmem.org/cgi/doi/10.1101//m.788008. pus damage for a simple visual discrimination problem that a separate group of rats could learn readily after hippocampus damage. Subsequently, some other studies confirmed Sara's finding that hippocampus damage causes retrograde, but not anterograde, amnesia in a serial feature-positive discrimination problem (Ross et al. 1984) and other arrangements of simple visual discrimination problems (Sutherland et al. 2001; Driscoll et al. 2004). The situation with respect to retrograde amnesia is more complex if one considers simple discriminations that may include stimulus modalities other than vision. No retrograde amnesia, temporally graded, and temporally ungraded retrograde amnesia have all been reported. For example, Kaut and Bunsey (2001) and Winocur and Mills (1970) found no reliable retrograde amnesia for preoperatively learned visual discriminations with hippocampus damage. Similarly, Mumby et al. (1999) did not find retrograde amnesia for two-choice object discriminations. In contrast, Wiig et al. (1996) report a temporally limited retrograde amnesia for object discriminations with fornix lesions in rats. Finally, Salmon et al. (1987) found retrograde amnesia that is not temporally graded or limited for objects after medial temporal damage that includes the hippocampus, amygdala, and adjacent cortical regions in monkeys. It is perhaps noteworthy that objects not only have visual features but also have a constellation of other surface properties that likely contribute to object perception and memory. Furthermore, contextual fear conditioning studies that have described temporally graded retrograde amnesia may have a strong odor component. In several studies, compounds such as ammonium hydroxide or acetic acid are added to the chambers and provide a potent nonvisual context cue (see Anagnostaras et al. 1999; Wiltgen et al. 2006). Other nonvisual cues such as the texture of the floor may also contribute to memory formation in contextual fear conditioning. It may be that a good deal of the variability in retrograde amnesia is related to the degree to which nonvisual information is available 
and, obviously, the extent to which damage includes structures other than the hippocampus. However, the type of memory being recalled should not be discounted, as this may be an additional factor that explains the variability of retrograde amnesia.

At present it is unclear whether the aforementioned reports of retrograde deficits in simple visual discrimination tasks represent anomalous results or reflect a memory process for which hippocampal function is generally relevant. In this light, we designed a series of experiments to systematically examine retrograde amnesia for simple visual discriminations after hippocampus damage. In the experiments reported below, we used a twochoice picture discrimination task in which nonvisual features cannot contribute to the discrimination. The procedure involved the simultaneous presentation of two pictures on computer monitors that were positioned side-by-side at one end of a trapezoidal-shaped pool. Swimming to one of the two pictures reliably enabled escape from the water onto a hidden platform that was submerged slightly below the surface of the water (Fig. 1A). Rats were first trained to solve picture discriminations, and, after various intervals, they received hippocampal damage, which was followed by retesting on the original and new picture discriminations.

The phenomenon of retrograde amnesia for picture discriminations gives us a novel opportunity to evaluate the systems

A

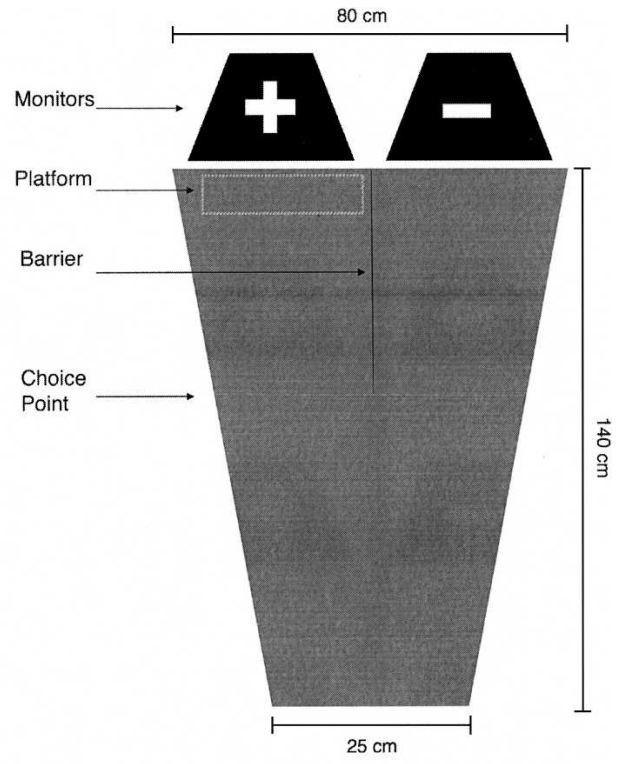

B

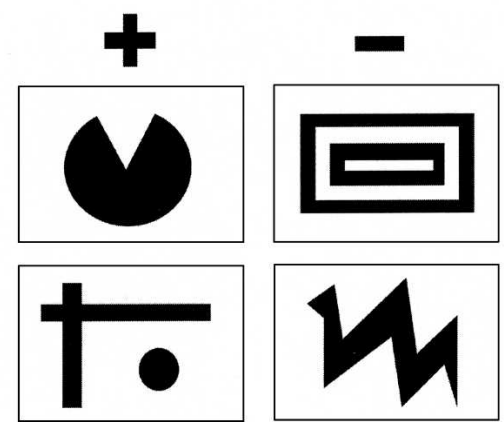

Figure 1. (A) Visual water task apparatus. A trapezoidal pool filled with water was used. A $60-\mathrm{cm}$-long barrier divided the pool into two alleys. Two 17-inch computer monitors were positioned at the wide end of the pool. (B) Sample pictures used for the visual discrimination task. consolidation hypothesis. On this hypothesis, memories that initially depend upon hippocampal circuitry can gradually acquire an independent representation in circuitry outside the hippocampus over a period of weeks or months. The view that the hippocampus is only temporarily essential to memories is supported by experiments using rats that have a systematically varied time interval between a learning episode and hippocampal damage. It has been reported that remote memories are unaffected by retrograde amnesia after hippocampal damage (Kim and Fanselow 1992; Kim et al. 1993; Maren et al. 1997; Anagnostaras et al. 1999; Winocur et al. 2001; Clark et al. 2002; Ross and Eichenbaum 2006). Others, however, have reported that the hippocampus is necessary even for the retrieval of remote memories (Bolhuis et al. 1994; Mumby et al. 1999; Sutherland et al. 2001; Clark et al. 2005; Martin et al. 2005; Lehmann et al. 2007). The latter experiments tend to rely on measuring memories involving spatial navigation or spatial contexts. There is a longstanding view that the hippocampus plays a special role in spatial memories (O'Keefe and Nadel 1978). Thus, our present experiments involving picture memories present a new opportunity to compare retrograde amnesia for recent and remote memories in a nonspatial memory task. In a further experiment, we investigated the relative effects of hippocampus damage on performance by rats that had mastered a one-trial picture discrimination task. The results of these experiments confirm that, in the intact rat over a wide range of learning parameters, the hippocampus plays an essential role in supporting recent and remote picture memories.

\section{Results}

\section{Retrograde amnesia for recent and remote picture memories}

Rats were trained consecutively on three different two-choice picture discriminations. Preoperatively, all rats rapidly mastered the basic escape procedure and achieved accuracies at or above $90 \%$ on all three picture discriminations. An ANOVA on the number of trials that were required to reach $90 \%$ accuracy on each of the three problems revealed a main effect of problem, $F_{(2,39)}=22.70, P<0.001$, and post hoc LSD test showed that the third problem was reliably acquired in fewer trials than either of the first two problems $(P<0.001)$. Acquisition rates for the first and second problems did not differ significantly from one another $(P>0.5)$. For each rat, performance on all three discrimination problems was maintained at or above $90 \%$ accuracy for at least three training sessions before surgery was performed (Fig. 2A).

We sought to determine if hippocampal damage produced retrograde amnesia for picture discriminations and whether recent and remote picture memories were differentially sensitive; thus, we varied the interval between the end of discrimination training and surgery. We evaluated rats that received surgery 1 (recent), 30 (intermediate), or 60 (remote) days after they completed discrimination training. Each rat was given one testing session for each of the three discrimination problems with the order counterbalanced. Then, we retrained the rats on the same discriminations until they were able to reach the criterion of $90 \%$ correct on each discrimination.

Postoperatively, at all three training-surgery intervals the sham surgery group demonstrated excellent retention of the discriminations, displaying better than $90 \%$ accuracy in each of the three problems in the first retention session. Rats with hippocampal damage exhibited severe retrograde amnesia for the picture discriminations. During the first postoperative testing session (Fig. 2B), rats with hippocampus damage performed at nearchance levels on all three problems. ANOVAs confirmed that, 

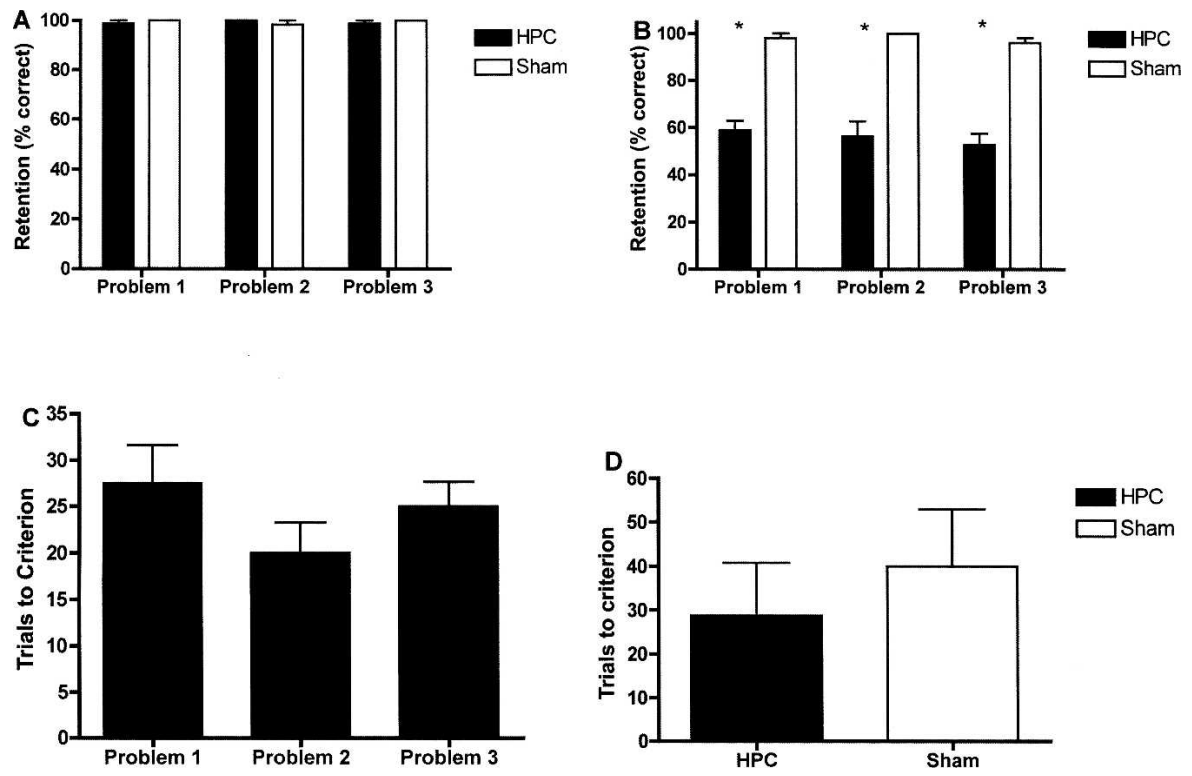

Figure 2. (A) Preoperative performance: Percent correct achieved during the final training session for each of the three visual discriminations is shown. (B) Postoperative performance: Retention of the three preoperatively acquired visual discriminations in rats with hippocampus damage or sham-operated controls is shown. Hippocampus-damaged rats were impaired on retention of all three discriminations. ${ }^{*} P<0.05$. (C) Reacquisition performance: Rats with hippocampus damage were able to reacquire al three previously learned acquisitions. $(D)$ Novel discrimination performance: Rats with hippocampus damage were able to acquire a novel visual discrimination. There was no significant difference in the rate of acquisition of a novel discrimination between hippocampus-damaged rats and sham-operated rats. All panels represent mean + SEM.

compared with sham lesion rats, rats with hippocampus damage made significantly more errors during the first postoperative session (10 trials) for each of the three training-surgery intervals, $F_{\text {remote (1,12) }}=48.2, P<0.001, F_{\text {intermediate }(1,12)}=36.0, P<0.001$, and $F_{\text {recent }(1,12)}=54.1, P<0.001$. There was no evidence of a temporal gradient in retrograde amnesia. An ANOVA confirmed that the deficit was equally severe for all three training-surgery intervals, $F_{(2,21)}=0.4, P=0.691$.

To determine if hippocampus damage blocks picture discrimination learning in the present task, we evaluated the rats' ability to relearn all three of the original discrimination problems. The hippocampus-damaged rats were able to relearn all three problems, and no differences in the rate of acquisition of the three different problems were observed (Fig. 2C), $F_{(2,21)}=1.26, P=0.305$. Finally, we assessed all rats' abilities to learn a new picture discrimination and found that there were no differences in the number of trials to achieve criterion on the new problem between rats with hippocampus damage and the rats of the sham lesion group, $F_{(1,12)}=0.40, P=0.540$ (Fig. 2D). There is also no evidence that the rats with hippocampal damage relearn the original picture problems in fewer trials than they learn the new picture problem.

\section{Retrograde amnesia for a well-learned simple visual discrimination and discrimination learning set}

Rats that are trained on successive simple discrimination problems typically learn to solve the discriminations more rapidly as they gain experience performing the task. This phenomenon, which is known as discrimination learning set, suggests that in addition to learning about the specific discriminative stimuli, animals also learn a general underlying rule or schema that supports very rapid solutions of new problems of the same class. We considered the possibility that the hippocampus damage may produce retrograde amnesia for the specific pictures used in the discriminations but that the rule or schema underlying picture discrimination learning set may be represented in memory independently of the hippocampus. Such an idea has been suggested recently by Tse et al. (2007). By training rats to solve the two-choice picture discrimination problem with novel pairs of pictures in each session to a stringent criterion, we were able to evaluate the effects of hippocampal damage on memories supporting learning set formation. To replicate the effects of hippocampus damage on a welllearned picture discrimination problem, prior to surgery all rats also were required to master a picture discrimination that involved a constant pair of pictures.

All rats in this experiment were trained on two different discrimination problems. One problem involved a pair of pictures that was constant across all training sessions (constant). The other problem involved two novel visual patterns each session (novel). Hippocampus damage was induced either 1 or $30 \mathrm{~d}$ following the completion of discrimination training.

\section{One-day training-surgery interval}

The constant and novel discrimination problems were rapidly acquired and retained with a high degree of accuracy by all rats. Many rats were able to simultaneously perform the constant component at $100 \%$ per session and at the same time demonstrate one-trial learning on the novel acquisition component. All rats achieved the criterion of $90 \%$ accuracy on both problems (10 trials per problem per session) above for both the constant and novel problems and maintained this level for at least three sessions prior to surgery. During the final three sessions, we analyzed performance on the first trial of each novel acquisition to ensure that the highly accurate performance could be attributed to rapid learning and not to an unaccounted artifact. We averaged the performance on the first trial across the final three sessions and found that rats made a correct choice $48.1 \%$ of the time. This corresponds to chance performance on the task and therefore demonstrates that rats were in fact demonstrating one-trial learning on the novel discriminations. On the final training session prior to surgery, all rats performed the constant problem without error (10 of 10). In the same session, rats in both groups required only 9.5 and 9.7 trials, respectively, to achieve nine correct responses out of 10 attempts on the novel discrimination problem. An ANOVA confirmed that the performances of the two groups did not differ prior to surgery on the novel problem $\left(F_{(1,15)}=0.48, P=0.50\right)$, and neither group committed any errors on the constant problem.

During the first postoperative session, all of the sham surgery rats performed the constant pattern discrimination problem without committing any errors. In contrast, during the first postoperative session, rats that had hippocampal damage were accurate on only $59 \%$ of their choices. An ANOVA confirmed that the postoperative difference in retention of the constant problem between the rats that received sham surgery and those that had hippocampus damage was statistically significant, $F_{(1,15)}=24.20$, $P<0.001$ (Fig. 3A).

During the first postoperative test session, rats with hippocampus damage were also impaired relative to sham surgery rats 

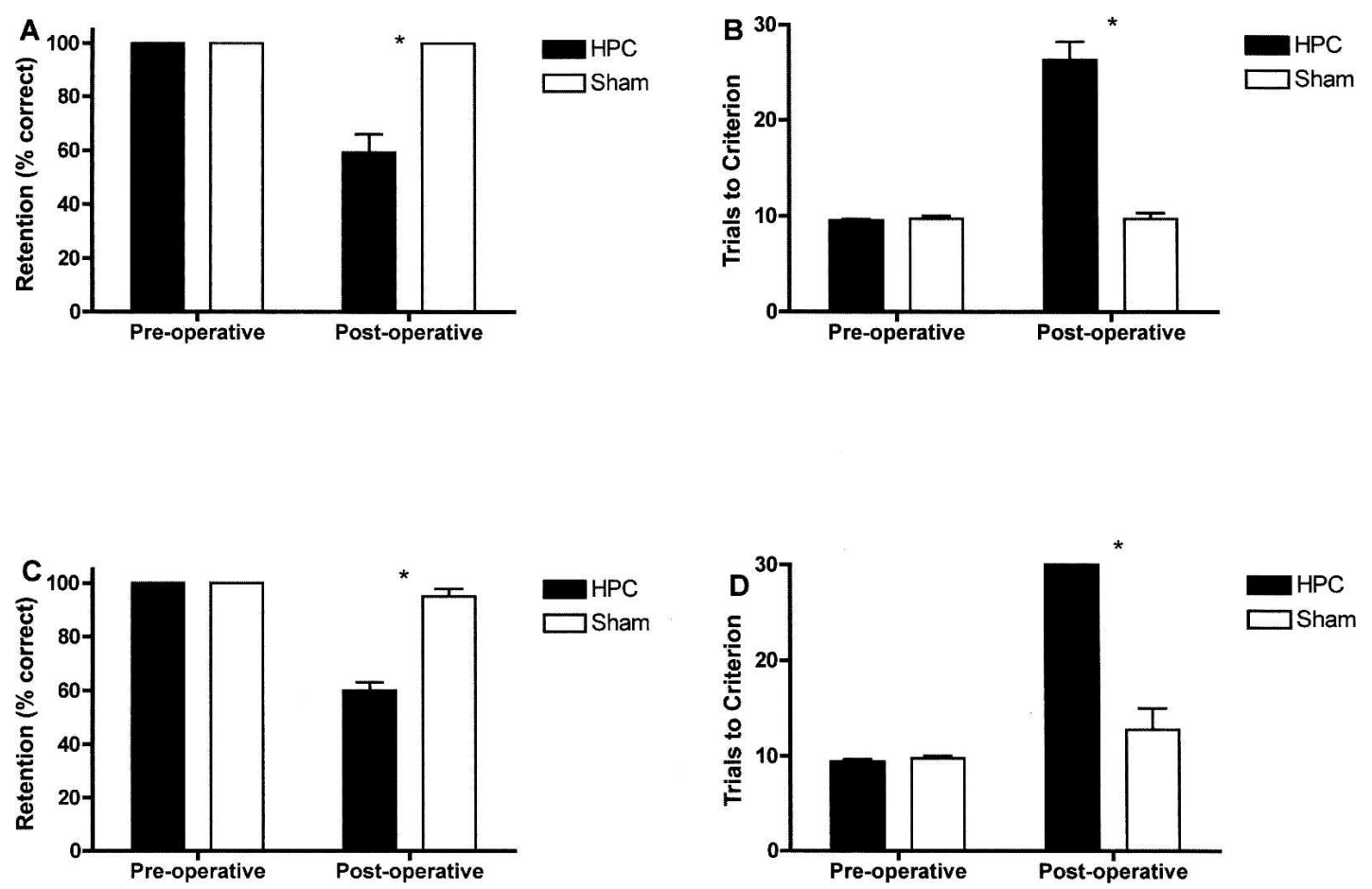

Figure 3. (A) Hippocampus damage resulted in impaired retention of the constant visual discrimination problem. The final preoperative training session and first postoperative test session with a 24-h training-surgery interval are shown. (B) There was also an initial impairment in acquisition of the daily novel discrimination. The final preoperative and first postoperative sessions with a 24-h training-surgery interval are shown. (C) Hippocampus damage resulted in impaired retention of the constant visual discrimination problem. The final preoperative training session and first postoperative test session with a 30-d training-surgery interval are shown. $(D)$ There was also an initial impairment in acquisition of the daily novel discrimination. The final preoperative and first postoperative sessions with a 30-d training-surgery interval are shown. All panels represent mean + SEM. ${ }^{\star} P<0.05$.

on a novel problem that they had never encountered previously, $F_{(1,15)}=49.17, P<0.001$. The sham surgery rats efficiently acquired the novel problem requiring only 9.7 trials to reach the criterion of nine correct responses out of 10 consecutive attempts, but HPC rats performed poorly, requiring 26.3 trials on average to achieve the criterion (Fig. 3B).

The effects of hippocampus damage on both the constant and novel discriminations were overcome by additional training. The hippocampus-damaged rats required 76 trials to meet the preoperative discrimination criterion of nine correct responses out of 10 attempts; postoperatively, they required 43 trials to reach criterion. The constant problem took significantly more trials $\left(F_{(1,18)}=11.08, P=0.004\right)$ to acquire preoperatively than it did to reacquire the same problem in the absence of the HPC.

\section{One-month training-surgery interval}

The pattern of postoperative impairment observed in rats that received surgery $30 \mathrm{~d}$ following the final discrimination training session was very similar to that described above for rats that had surgery $24-\mathrm{h}$ after discrimination training. All rats showed excellent preoperative performance on both the acquisition and retention components. During the final preoperative session, the group to receive hippocampus damage scored $100 \%$ correct on the constant problem and acquired the novel problem in 9.4 trials while the to-be-sham group also scored 100\% correct and required 9.8 trials to reach criterion on the novel problem. No significant effect of group was identified prior to surgery for acquisition of the novel problem $\left(F_{(1,7)}=0.98, P=0.356\right)$, and neither group made an error during the final preoperative session for the retention problem.

More than 1 mo after last performing the task, rats that underwent sham surgery showed no significant changes in per- formance from their preoperative level on the novel discrimination $\left(F_{(1,6)}=1.76, P=0.233\right)$ or the constant discrimination $\left(F_{(1,6)}=3.00, P=0.134\right)$. However, rats with hippocampus damage were impaired relative to sham surgery rats on both the constant (Fig. 3C) and the novel discriminations (Fig. 3D). During the first postoperative testing session, sham rats acquired the novel problem in 12.8 trials. Rats with HPC damage were not able to acquire the novel problem within the maximum number of 30 trials. During the same testing session, the HPC-damaged rats showed poor retention of the constant problem (60\%) compared with the performance of $95 \%$ correct achieved by rats that had undergone sham surgery. The between-group difference in the number of trials required to achieve criterion on the novel discrimination was significant $\left(F_{(1,7)}=76.19, P<0.001\right)$, as was the effect of group on retention of the constant discrimination $\left(F_{(1,7)}=63.52, P<0.001\right)$.

Finally, the amount of time that passed between preoperative discrimination training and hippocampus damage did not affect performance on the novel discrimination $\left(F_{(1,13)}=1.79\right.$, $P=0.204)$ or the constant discrimination $\left(F_{(1,13)}=0.01\right.$, $P=0.923)$. Thus, the second experiment also provided no evidence for a temporally graded retrograde amnesia for simple visual discrimination learning nor was there evidence for sparing of the rule or schema supporting the learning set.

Rats that sustained hippocampus damage $30 \mathrm{~d}$ after the completion of training were able to relearn the discrimination problems with further postoperative training. The mean numbers of trials required after surgery for rats with hippocampus damage to reach the criterion were 32.0 for the constant discrimination and 45.8 for the novel discrimination problem. The number of trials needed to re-attain criteria was equivalent in the two groups with hippocampus damage tested at the two training- 
surgery time points both for the constant discrimination $\left(F_{(1,13)}=0.79, P=0.399\right)$ and the novel discrimination $\left(F_{(1,13)}=2.29, P=0.154\right)$.

\section{Relationship between spared hippocampus volume and retrograde amnesia}

The experiments presented thus far demonstrate that the intact hippocampus plays an essential role in memories that support simple two-choice picture discrimination problems. Thus, in rats that learn a simple picture discrimination and subsequently undergo hippocampus lesion surgery, one would expect the severity of retrograde amnesia to be associated with the volume of hippocampus damaged. To test this prediction, we trained a group of rats on a single two-choice picture discrimination problem and then created hippocampus lesions either by injecting NMDA at seven injection sites bilaterally in the hippocampus or made smaller hippocampus lesions by injecting NMDA at five bilateral sites in the hippocampus (see Methods). We then statistically evaluated the relationship between the severity of retrograde amnesia for the picture discrimination and the amount of spared hippocampal tissue.

Lesions made with five bilateral NMDA injections spared $\sim 33.9 \% \pm 4.1 \%$ of the total hippocampal volume (Fig. 4). In

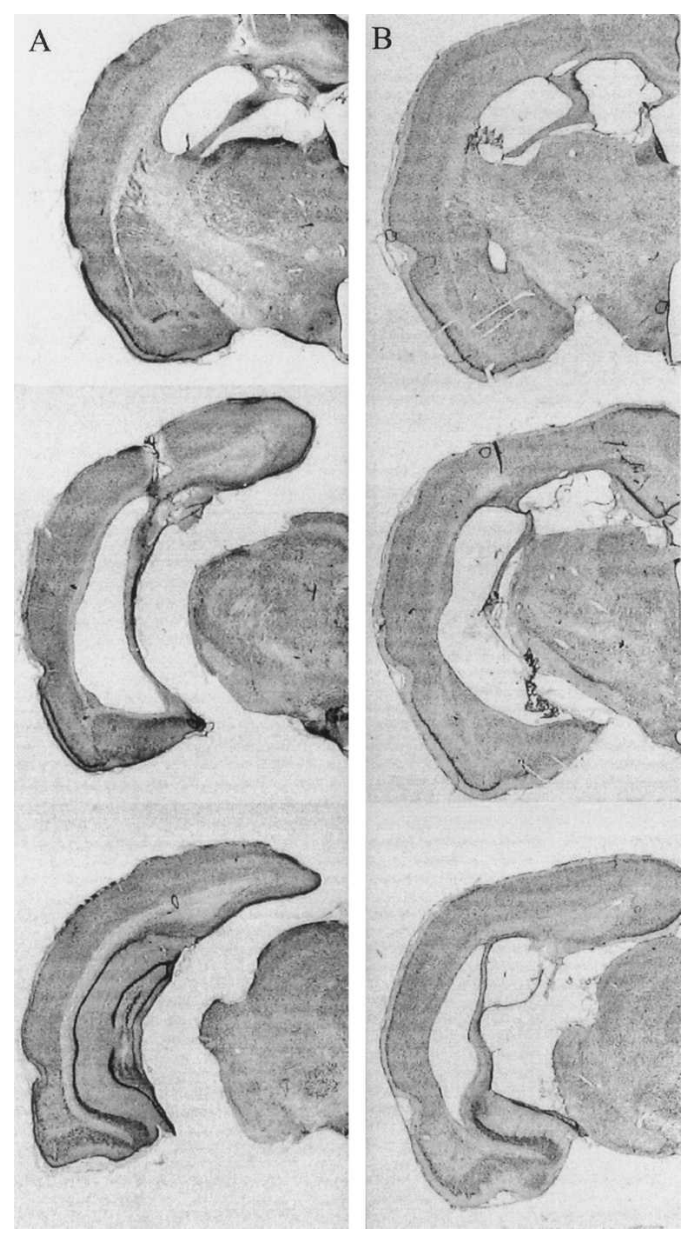

Figure 4. Cresyl Violet-stained sections showing NMDA-induced damage throughout the extent of the hippocampus. (A) Typical damage caused by injecting NMDA at five sites per hemisphere. On average, $34 \%$ of the HPC (mostly ventral HPC) was spared. (B) Typical damage resulting from seven injections of NMDA per hemisphere. On average, only $21 \%$ of the hippocampus remains.
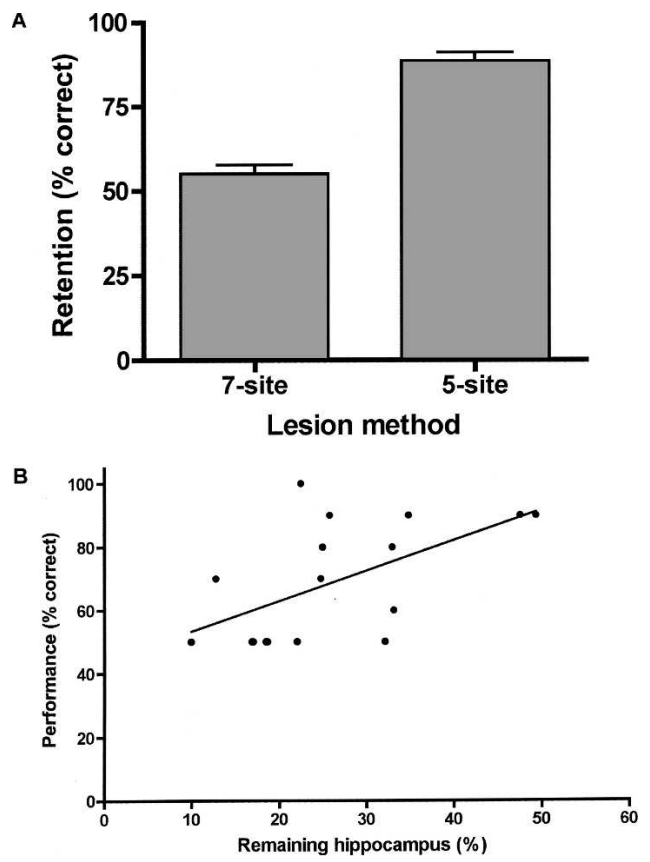

Figure 5. (A) Retention of a single visual discrimination problem in rats with larger hippocampal lesions produced with seven injections of NMDA per hemisphere and lesions produced with five injections per hemisphere. Larger lesions produced significantly worse retention of the discrimination. Data represent mean + SEM during the first postoperative testing session. (B) Correlation of retention during the first postoperative testing session with the amount of remaining hippocampal tissue. There was a significant positive relationship between the amount of hippocampus tissue remaining and retention of a single discrimination.

comparison, a lesion produced with seven bilateral injections of NMDA spared only $20.5 \% \pm 2.4 \%$ of the hippocampus. An ANOVA demonstrated a significant group difference in spared hippocampus volume $\left(F_{(1,15)}=9.05, P=0.009\right)$. The postoperative performance of rats with five-injection-site lesions was compared to the rats with seven-injection-site lesions (Fig. 5A). An ANOVA showed that seven-site lesions resulted in significantly more retrograde amnesia for the visual discrimination than did five-site lesions $\left(F_{(1,15)}=74.39, P<0.001\right)$. A significant positive correlation was found between lesion volume and retrograde amnesia, such that larger lesions were associated with poorer postoperative retention of the discrimination, $r=0.571, P=0.017$ (Fig. 5B).

\section{Discussion}

The results presented here confirm the existence of severe retrograde amnesia for picture discriminations. Furthermore, it is confirmed that even though the hippocampus is not necessary to resolve picture discriminations, if the discriminations are resolved while the hippocampus is intact, they are vulnerable to subsequent hippocampus damage and remain vulnerable for at least 60-80 d after they have been learned. These findings imply that the hippocampus normally participates in the long-term retention of memories supporting simple picture discriminations.

In the first experiment, we trained rats on three discriminations and assessed the effects of hippocampus damage on rats' retentions of these discriminations. Different intervals between discrimination training and surgery were studied; recent (1-20 d), intermediate (20-30 d), and remote (60-80 d). Postoperatively, animals with hippocampus damage displayed severe ret- 
rograde amnesia for the discriminations, regardless of the training-surgery interval, whereas rats with intact hippocampi demonstrated excellent retention. However, postoperative retraining on the original discriminations as well as training on a novel discrimination revealed a spared ability to learn simple picture discriminations.

A further experiment evaluated both retention of a welllearned discrimination and the ability to demonstrate one-trial picture discrimination learning (learning set). Again, regardless of the interval between training and surgery ( 1 or $30 \mathrm{~d}$ ), severe retrograde amnesia for the constant discrimination was apparent postoperatively. Initially, rats with hippocampus damage were poor at learning new discriminations. However, after a few additional postoperative training sessions the rats with hippocampus damage recovered their ability to demonstrate rapid withinsession acquisition of novel discriminations. This result shows that after hippocampus damage, the win-stay rule that had been learned preoperatively (as demonstrated by one-trial learning of new discriminations) had to be relearned.

Finally, we demonstrated by varying the extent of the hippocampal lesions that the severity of retrograde amnesia was positively correlated with the volume of damaged hippocampus; partial sparing of the hippocampus was associated with better postoperative retention of the picture discrimination. Very similar relationships between the extent of hippocampus damage and the severity of retrograde amnesia in contextual fear conditioning and spatial navigation have already been demonstrated. This highlights the importance of ensuring that hippocampus damage is sufficiently extensive if inferences about hippocampal independence of memories are to be valid.

One aim of the experiments described here was to determine whether recent and remote picture memories are equally disrupted by hippocampus damage. Our results demonstrate that NMDA-induced hippocampus lesions that followed discrimination training caused severe retrograde amnesia. However, we observed no evidence of a temporal gradient associated with the retrograde amnesia. Even with quite long intervals between discrimination training and surgery, complete retrograde amnesia for the discrimination was apparent. Thus, the hippocampus appears to play a long-term role in retention of memories supporting picture discriminations. Although this finding conflicts with the standard model of memory consolidation (e.g., Squire et al. 1984), the findings of the present study are consistent with previous studies that point to a long-term role of the hippocampus in memory storage. For example, Sutherland and colleagues demonstrated that rats trained on two water maze tasks had retrograde amnesia for both recent and remote memories following hippocampus damage (Sutherland et al. 2001). Hippocampus damage following training on a cross maze has also been shown to produce retrograde amnesia with equal severity at $2 \mathrm{wk}$ and 9 mo (Winocur et al. 2005). Similarly, Bolhuis and colleagues (1994) observed no temporal gradient for retrograde amnesia in rats that were trained in a spatial water maze task $14 \mathrm{wk}$ prior to HPC damage. Therefore, the results of the present study join with others showing that at least some memory types are dependent on the hippocampus for long-term storage.

It seems unlikely that we did not allow a long enough interval between discrimination and surgery to allow betweensystem memory consolidation to occur. Clarke et al. have demonstrated using a social transmission of food preference test that 1-d-old memories are vulnerable to hippocampus damage but 30-d-old memories are spared (Clark et al. 2002). Similarly, temporally graded retrograde amnesia has been shown for contextual fear conditioning with remote memory training occurring $28 \mathrm{~d}$ prior to hippocampus damage (Anagnostaras et al. 1999). Other studies have demonstrated that memories were spared if behav- ioral training occurred just a few days prior to hippocampus damage (Winocur et al. 2001; Tse et al. 2007). However, we evaluated retention across intervals ranging from 1 to $60 \mathrm{~d}$ in the present study and observed no evidence of an association between the severity of retrograde amnesia after hippocampus damage and the age of the memory at the time of the damage.

These data may also be problematic for Multiple Trace Theory (Nadel and Moscovitch 1997). In their view, context-rich, episodic memories may depend upon hippocampus regardless of whether they are recent or remote. After a temporary interval of hippocampal dependence, more gist-like, semantic memories can be retrieved independently of the hippocampus. At face value, simple visual discriminations should be squarely within the latter category, since there is a single consistent set of stimulus attributes that always predict the reinforcer. Contrary to the prediction of multiple trace theory, there is no evidence for sparing of remote picture memories in our task. From our demonstration that there is no anterograde effect of hippocampal damage in the same task, it is clear that a non-hippocampal network can resolve the discrimination. Combined lesion experiments suggest that this network involves cortico-striatal circuitry (Sutherland et al. 2007). Why does this non-hippocampal network not acquire a useful independent memory of the pictures when the hippocampus is intact, either through its own associative processes acting in parallel with those of the hippocampus or through systems consolidation? There are at least two possibilities that cannot be distinguished by the available evidence: competition and interference. Several theorists have suggested that the hippocampal system forms a complex configural/ conjunction representation of cues comprising contexts or learning episodes but that there are non-hippocampal memory networks that can represent simpler features or cue elements. Either configural/conjunctive or elemental representations can participate in learned associations. In order to explain the severe retrograde amnesia without anterograde amnesia in contextual fear conditioning with hippocampal damage, Maren et al. (1997) suggest that the hippocampal system enters into a competition with other systems and further that the hippocampus normally dominates. This hypothetical process is consistent with results not only from contextual fear conditioning, but from the present picture memory task as well (see also Driscoll et al. 2005). The second possibility involving interference is more speculative. Perhaps hippocampal and non-hippocampal networks both record information from learning episodes. However, if their contributions were largely in parallel, then removal of the hippocampus would not yield severe retrograde amnesia. Instead, we propose that hippocampal output significantly modifies the activity in regions that represent retrieval cues. Normally, these cues can access hippocampal and non-hippocampal networks and thereby participate in configural/conjunctive and elemental associations. If after learning, the hippocampus is removed and the hippocampal output lacking, then retrieval cues do not create the familiar pattern of activity in relevant representational networks and non-hippocampal memories are not contacted. A further implication is that if either of the competition or interference accounts is correct, then the hippocampus will have a contribution to memory tasks that is not limited to those that can be formally described as configural/conjunctive or episodic. Instead, via the competition or interference process the hippocampus makes essential contributions to tasks that are solvable by elemental learning systems, including simple discriminations or more procedural memory tasks. Recent evidence has suggested that the unique anatomical properties of the hippocampus provide a mechanism for minimizing interference (Becker 2005), possibly explaining why the hippocampus plays a role in a wide range of memory types. 
The results of these experiments confirm the initial findings of Sara (1981) that hippocampus damage causes retrograde amnesia for simple picture discriminations. We have further extended these findings to show that even with long trainingsurgery intervals, hippocampus damage disrupts retention of simple picture memories. However, we find that in the absence of the hippocampus these same discriminations can be acquired and stored normally. Thus, the hippocampus is involved in the acquisition and long-term storage of simple discrimination learning even though it is not required for this type of learning. Thus, we show here that the hippocampus normally plays a long-term role in the storage of a wider range of memory types than currently predicted.

\section{Methods}

\section{Subjects}

Subjects were 57 male Long-Evans rats obtained from Charles River Laboratories (Quebec, Canada). All rats were between 300 and $400 \mathrm{~g}$ at the start of testing. They were pair-housed in standard polyurethane cages. Temperature and humidity were held constant in the housing room, and lights were maintained on a 12-h light/dark cycle. All training took place during the light phase. Rats were provided with ad libitum access to rat chow and water. All experiments were conducted in accordance with the Canadian Council on Animal Care guidelines.

\section{Apparatus}

All visual discrimination experiments were carried out in the visual water task (Prusky et al. 2000, 2004). The apparatus (Fig. 1A) was a trapezoidal stainless steel pool $(140 \mathrm{~cm}$ long, $25 \mathrm{~cm}$ wide at the narrow end, and $80 \mathrm{~cm}$ at the wide end) filled with water. The widest end of the pool was constructed of clear Plexiglas, behind which two computer monitors were placed. A $60-\mathrm{cm}$ barrier divided one end of the pool into two alleys with one monitor visible from each side of the barrier. The monitors displayed black and white images of geometrical patterns (Fig. 1B), one of which $(\mathrm{S}+)$ was paired with a hidden platform that allowed escape from the pool. The position of the platform and S+ (i.e., left versus right side of the pool) followed a pseudo random pattern so that a spatial strategy could not be used to solve the discrimination. Rats were only removed from the pool once they climbed on the platform. Trials in which rats swam directly to the escape platform were marked as correct. If the rat first crossed the plane perpendicular to the edge of the barrier on the incorrect side, the trial was scored as incorrect. All training was conducted with all lights off such that the only light emitted in the room came from the two display monitors and a third experiment control monitor that was not visible from within the pool.

\section{Surgery}

An injection of diazepam $(20 \mathrm{mg} / \mathrm{kg})$ was given to each rat 10 min prior to anesthesia induction. Rats were then anesthetized with isofluorane ( $4 \%$ with $2 \mathrm{~L}$ per minute of oxygen) and maintained at $2 \%$ once a surgical plane was established. Rats were positioned in a stereotaxic instrument (David Kopf Instruments), and a midline incision was made in the scalp. Lesions were produced by injecting $0.5 \mu \mathrm{L}$ of NMDA $(10 \mathrm{mg} / \mathrm{mL})$ through 30 gauge cannulae, at multiple bilateral sites. The infusion sites were located $-3.1,-4.1,-5.0,-5.3$, and $-6.0 \mathrm{~mm}$ posterior to bregma, $\pm 1.5, \pm 3.0, \pm 3.0, \pm 5.2$, and $\pm 5.0 \mathrm{~mm}$ lateral of midline, and $-3.6,-4.0,-4.0,-7.0$, and $-7.0 \mathrm{~mm}$ ventral to the skull surface. In one experiment, in order to create more complete hippocampal lesions, one group of rats was given seven bilateral NMDA injections at sites located $-3.1,-4.1,-5,-5$, $-5.8,-5.8$, and $-5.8 \mathrm{~mm}$ posterior to bregma, $\pm 1.5, \pm 3.0$ $\pm 3.0, \pm 5.2, \pm 4.4, \pm 5.1$, and $\pm 5.1 \mathrm{~mm}$ lateral of midline, and $-3.6,-4.0,-4.0,-7.3,-4.4,-7.5$, and $-6.2 \mathrm{~mm}$ ventral to the surface of the skull. An infusion rate of $0.125 \mu \mathrm{L} / \mathrm{min}$ was used, and the cannulae remained in place for an additional $4 \mathrm{~min}$ after infusion to allow NMDA to diffuse away from the cannulae tips. The scalp was closed with dissolvable suture material (Vicryl $3-0)$. All rats were administered diazepam $(20 \mathrm{mg} / \mathrm{kg})$ upon first signs of wakefulness, and additional doses were given as needed to suppress seizure activity associated with NMDA injection. Control rats received scalp incisions and sutures, but no microinjections of NMDA were made. All rats received buprenorphine $(0.05 \mathrm{mg} / \mathrm{kg})$ following surgery to alleviate pain. A postoperative recovery period of $1 \mathrm{wk}$ was allowed for all rats before behavioral testing commenced.

\section{Behavioral procedures}

Retrograde amnesia for simple visual discriminations and temporal effects Rats were trained on three different two-choice simple visual pattern discriminations. On days $1-20$ rats received 10 daily trials on the first discrimination. A 10-d break in which no training took place was given between each different discrimination problem. On days 31-50 the same rats were trained on the second discrimination problem, and on days 61-80 they were trained on the final discrimination problem. Each daily session consisted of 10 discrimination trials. Rats were trained in squads of three so that the inter-trial interval was $\sim 1 \mathrm{~min}$.

Hippocampal damage $(n=8)$ or sham surgery $(n=6)$ was performed $24 \mathrm{~h}$ after the final training session on the third discrimination. Postoperative testing began $1 \mathrm{wk}$ following surgery. Each rat was tested for retention of all three preoperatively acquired picture discriminations. During each daily session, rats were trained on only one of the three discrimination problems, and the order of testing was counterbalanced such that some rats were exposed to the most remote discrimination first and others to the most recent discrimination. Testing continued until the $90 \%$ correct criterion was achieved for each picture discrimination. Then, rats were trained on a novel visual pattern discrimination until performance was $90 \%$ correct or greater per session for three consecutive sessions.

\section{Retrograde amnesia for a well-learned simple visual discrimination versus rapid acquisition of a new discrimination}

To compare the effects of hippocampus damage on the retention of a well-learned visual pattern discrimination to its effects on the ability to rapidly acquire novel visual pattern discriminations, rats were trained on a multiple-component adaptation of the visual discrimination task. One component of the task (constant) involved a constant pair of pictures that were used in every session. The second component of the task (novel) involved training rats on a novel discrimination each session. First, rats were trained daily just on the constant discrimination until they reached a criterion of $80 \%$ accuracy in 10 trials. Then, the novel discriminations were introduced and the daily training sessions consisted of five trials on the fixed problem followed by training on a novel discrimination until nine correct choices in 10 successive trails was achieved or until 30 trials were completed, whichever came first. After the novel discrimination trials were completed, the session ended with five further trials on the constant discrimination. Preoperative daily training sessions were conducted until rats could perform the constant discrimination at $90 \%$ accuracy or better and also perform the novel discrimination at a level of nine out of 10 consecutive trials correct within 12 trials (nine trials would be the minimum number of trials possible, and 30 was used as the maximum number of trials). This took between 10 and 15 training sessions to achieve. HPC damage (or corresponding sham surgery) was induced either $1 \mathrm{~d}\left(n_{\mathrm{HPC}}=10, n_{\text {sham }}=7\right)$ or $30 \mathrm{~d}\left(n_{\mathrm{HPC}}=5, n_{\text {sham }}=4\right)$ after the completion of training. One week after surgery, testing sessions were conducted following the same regimen that was used during the terminal preoperative sessions.

\section{Relationship between spared hippocampus volume and anterograde amnesia}

To determine whether the extent of hippocampus damage affects the postoperative retention for picture memories we utilized two 
NMDA injection protocols to produce lesions of different sizes. Rats were trained on a single two-choice visual pattern discrimination for 20 consecutive days. Each day the rats received a single training session that consisted of 10 trials. During this training period, all rats achieved a criterion of nine out of 10 trials correct for at least the last three sessions. Hippocampal damage was induced $24 \mathrm{~h}$ after the final discrimination training session. Lesions were made in the hippocampus by injecting NMDA at either five $(n=7)$ or seven $(n=10)$ sites per hemisphere (see Surgery). After a 1 -wk recovery period, rats were tested on the visual pattern discrimination task that they had been trained on preoperatively.

\section{Tissue quantification}

Remaining HPC volumes were calculated using the Cavalieri method. Sections were cut on a cryostat at a thickness of $50 \mu \mathrm{m}$ and stained with Cresyl Violet. A sampling fraction of $1 / 10$ was used, yielding an average of $\sim 11$ sections per HPC. Pictures were taken through the entire extent of the HPC using a QImaging Retiga CCD camera (Burnaby) attached to a Zeiss Axioscop 2 mot plus microscope with a $2.5 \times$ objective. Using the software program Image $(\mathrm{NIH})$ a systematic, random grid with an area per point of $0.5 \mathrm{~mm}^{2}$ was thrown over each image. All points contacting the HPC were counted, and total volume was calculated.

A mean baseline HPC volume was calculated using six control rats. To calculate the percentage of HPC tissue remaining in a given lesion in a rat, the volume was taken as a percentage of the aforementioned baseline.

\section{Acknowledgments}

Financial support for these experiments was provided by AHFMR and NIMH (grant MH61460 to R.J.S.).

\section{References}

Alvarado, M.C. and Rudy, J.W. 1995. Rats with damage to the hippocampal-formation are impaired on the transverse-patterning problem but not on elemental discriminations. Behav. Neurosci. 109: 204-211.

Anagnostaras, S.G., Maren, S., and Fanselow, M.S. 1999. Temporally graded retrograde amnesia of contextual fear after hippocampal damage in rats: Within-subjects examination. J. Neurosci. 19: $1106-1114$.

Becker, S. 2005. A computational principle for hippocampal learning and neurogenesis. Hippocampus 15: 722-738.

Bolhuis, J.J., Stewart, C.A., and Forrest, E.M. 1994. Retrograde amnesia and memory reactivation in rats with ibotenate lesions to the hippocampus or subiculum. Q. J. Exp. Psychol. B 47: 129-150.

Broadbent, N.J., Squire, L.R., and Clark, R.E. 2007. Rats depend on habit memory for discrimination learning and retention. Learn. Mem. 14: $145-151$.

Clark, R.E., Broadbent, N.J., Zola, S.M., and Squire, L.R. 2002. Anterograde amnesia and temporally graded retrograde amnesia for a nonspatial memory task after lesions of hippocampus and subiculum. J. Neurosci. 22: 4663-4669.

Clark, R.E., Broadbent, N.J., and Squire, L.R. 2005. Hippocampus and remote spatial memory in rats. Hippocampus 15: 260-272.

Driscoll, I., Sutherland, R.J., Prusky, G.T., and Rudy, J.W. 2004. Damage to the hippocampal formation does not disrupt representational flexibility as measured by a novelty transfer test. Behav. Neurosci. 118: $1427-1432$.

Kaut, K.P. and Bunsey, M.D. 2001. The effects of lesions to the rat hippocampus or rhinal cortex on olfactory and spatial memory: Retrograde and anterograde findings. Cogn. Affect. Behav. Neurosci. 1: $270-286$.

Kim, J.J. and Fanselow, M.S. 1992. Modality-specific retrograde amnesia of fear. Science 256: 675-677.

Kim, J.J., Rison, R.A., and Fanselow, M.S. 1993. Effects of amygdala hippocampus, and periaqueductal gray lesions on short- and long-term contextual fear. Behav. Neurosci. 107: 1093-1098.

Lehmann, H., Lacanilao, S., and Sutherland, R.J. 2007. Complete or partial hippocampal damage produces equivalent retrograde amnesia for remote contextual fear memories. Eur. J. Neurosci. 25: 1278-1286.

Maren, S., Aharonov, G., and Fanselow, M.S. 1997. Neurotoxic lesions of the dorsal hippocampus and Pavlovian fear conditioning in rats. Behav. Brain Res. 88: 261-274.

Martin, S.J., de Hoz, L., and Morris, R.G. 2005. Retrograde amnesia: Neither partial nor complete hippocampal lesions in rats result in preferential sparing of remote spatial memory, even after reminding. Neuropsychologia 43: 609-624.

McDonald, R.J., King, A.L., Wasiak, T.D., Zelinski, E.L., and Hong, N.S 2007. A complex associative structure formed in the mammalian brain during acquisition of a simple visual discrimination task: Dorsolateral striatum, amygdala, and hippocampus. Hippocampus 17: 759-774.

Mumby, D.G., Astur, R.S., Weisend, M.P., and Sutherland, R.J. 1999. Retrograde amnesia and selective damage to the hippocampal formation: Memory for places and object discriminations. Behav. Brain Res. 106: 97-107.

Nadel, L. and Moscovitch, M. 1997. Memory, consolidation, retrograde amnesia and the hippocampal complex. Curr. Opin. Neurobiol. 7: 217-227.

O'Keefe, J. and Nadel, L. 1978. The hippocampus as a cognitive map. Oxford University Press, London, UK.

Prusky, G.T., West, P.W., and Douglas, R.M. 2000. Behavioral assessment of visual acuity in mice and rats. Vision Res. 40: 2201-2209.

Prusky, G.T., Douglas, R.M., Nelson, L., Shabanpoor, A., and Sutherland, R.J. 2004. Visual memory task for rats reveals an essential role for hippocampus and perirhinal cortex. Proc. Natl. Acad. Sci. 101: $5064-5068$.

Ross, R.S. and Eichenbaum, H. 2006. Dynamics of hippocampal and cortical activation during consolidation of a nonspatial memory. $J$. Neurosci. 26: 4852-4859.

Ross, R.T., Orr, W.B., Holland, P.C., and Berger, T.W. 1984. Hippocampectomy disrupts acquisition and retention of learned conditional responding. Behav. Neurosci. 98: 211-225.

Salmon, D.P., Zola-Morgan, S., and Squire, L.R. 1987. Retrograde amnesia following combined hippocampus-amygdala lesions in monkeys. Psychobiology 15: 37-47.

Sara, S.J. 1981. Memory deficits in rats with hippocampal or cortical lesions: Retrograde effects. Behav. Neural Biol. 32: 504-509.

Squire, L.R., Cohen, N.J., and Nadel, L. 1984. The medial temporal region and memory consolidation: A new hypothesis. In Memory consolidation (eds. H. Weingartner and E. Parker), pp. 185-210. Lawrence Erlbaum, Hillsdale, NJ.

Sutherland, R.J. and Rudy, J.W. 1989. Configural association theory-the role of the hippocampal-formation in learning, memory and amnesia. Psychobiology 17: 129-144.

Sutherland, R.J., Weisend, M.P., Mumby, D., Astur, R.S., Hanlon, F.M., Koerner, A., Thomas, M.J., Wu, Y., Moses, S.N., Cole, C., et al. 2001. Retrograde amnesia after hippocampal damage: Recent vs. remote memories in two tasks. Hippocampus 11: 27-42.

Sutherland, R.J., Stone, J.C., and McDonald, R.J. 2007. Forgetting of picture memories in rats: A challenge to standard consolidation theory. Presentation 305.25. Neuroscience 2007, San Diego, November 3-7, 2007. San Diego, CA: Society for Neuroscience.

Tse, D., Langston, R.F., Kakeyama, M., Bethus, I., Spooner, P.A., Wood E.R., Witter, M.P., and Morris, R.G. 2007. Schemas and memory consolidation. Science 316: 76-82.

Whishaw, I.Q. and Tomie, J.A. 1991. Acquisition and retention by hippocampal rats of simple, conditional, and configural tasks using tactile and olfactory cues: Implications for hippocampal function. Behav. Neurosci. 105: 787-797.

Wiig, K.A., Cooper, L.N., and Bear, M.F. 1996. Temporally graded retrograde amnesia following separate and combined lesions of the perirhinal cortex and fornix in the rat. Learn. Mem. 3: 313-325.

Wiltgen, B.J., Sanders, M.J., Anagnostaras, S.G., Sage, J.R., and Fanselow, M.S. 2006. Context fear learning in the absence of the hippocampus. J. Neurosci. 26: 5484-5491.

Winocur, G.M. and Mills, J.A. 1970. Transfer between related and unrelated problems following hippocampal lesions in rats. J. Comp. Physiol. Psychol. 73: 162-169.

Winocur, G., McDonald, R.M., and Moscovitch, M. 2001. Anterograde and retrograde amnesia in rats with large hippocampal lesions. Hippocampus 11: 18-26.

Winocur, G., Moscovitch, M., Caruana, D.A., and Binns, M.A. 2005. Retrograde amnesia in rats with lesions to the hippocampus on a test of spatial memory. Neuropsychologia 43: 1580-1590.

Received October 1, 2007; accepted in revised form January 28, 2008. 


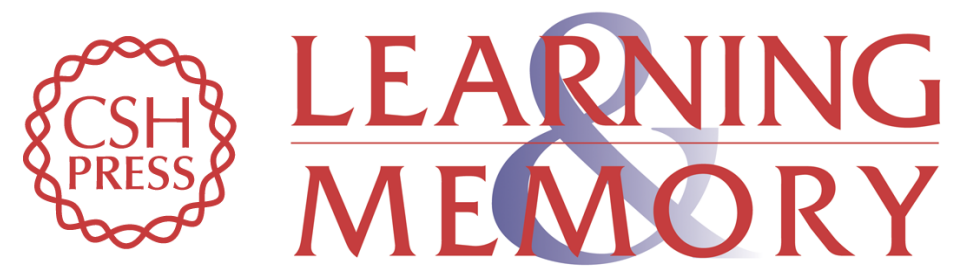

\section{Retrograde amnesia for visual memories after hippocampal damage in rats}

Jonathan Epp, Julian R. Keith, Simon C. Spanswick, et al.

Learn. Mem. 2008, 15:

Access the most recent version at doi:10.1101//m.788008

References This article cites 32 articles, 9 of which can be accessed free at:

http://learnmem.cshlp.org/content/15/4/214.full.html\#ref-list-1

License

Email Alerting Receive free email alerts when new articles cite this article - sign up in the box at the Service top right corner of the article or click here. 